\title{
Preliminary investigation on the feasibility of radiometric techniques to detect faults in buried cable joints
}

\author{
Demetres Evagorou, Kevin F. Goddard, Paul L. Lewin, and Steve G. Swingler \\ The Tony Davies High Voltage Laboratory \\ Electronics and Computer Science \\ University of Southampton \\ Southampton, Hampshire, SO17 1BJ, UK \\ Email: D.Evagorou@ soton.ac.uk
}

\begin{abstract}
This paper investigates the preliminary use of radiometric techniques to the detection of PDs in buried cables, and in particular to cable joints. The transfer function from the source to the detector is a function of the propagation characteristics of the media involved. In the case of radiometric detection the inclusion of soil, in general a lossy and dispersive medium with frequency and content dependent characteristics, further contributes to signal attenuation. The work undertaken here examines whether a repetitive pulse of varying amplitude and frequency, injected into an experimental arrangement that simulates buried power cables, is being detected by two simple antennae above ground. Successful detection of the pulses showed the preliminary possibility of the use of such techniques in PD detection, which creates the need for further experiments and antenna designs to be explored.
\end{abstract}

Index Terms-Partial Discharges; Radiometric Techniques; HF/VHF

\section{INTRODUCTION}

Partial Discharge (PD) measurements in cables and their accessories are an integral part of condition monitoring of High Voltage (HV) equipment. Cable joints are considered a weak point in the insulation system, and new techniques need to be developed in order to monitor them. This paper examines the use of airborne measurements in the vicinity of the joint, in order to detect the small electromagnetic pulse emitted by a discharging PD. This method does not require access to an earthing wire compared to inductive High (HF) or Very High Frequency (VHF) sensors. Moreover in cases where there is no access to the joints the sensor is placed on the substation side and in order for the pulse to reach the sensor it has to travel through a long section of the cable (a high frequency attenuator) resulting in practically being buried in background noise. The use of a radiometric technique could potentially resolve this problem, detection being done directly above the joint.

In order to tackle the above problem, as a first step, an experiment was designed based on a model of a cable trough to test two different scenarios using two simple antennae designs

This work is supported by the UK Engineering and Physical Sciences Research Council (EPSRC) under Grant EP/K021699/1 at ground level. Traditionally airborne ultrasonic sensors have been used mainly to monitor transformers due to the lower attenuation of sound waves in oil. Recently radio frequency antennae have been employed to monitor open-air substations with Ultra High Frequency characteristics (UHF). The propagation of the signal through soil, a dispersive medium with frequency dependent attenuation required the antennae designed to operate in the High and Very High Frequency (VHF) ranges.

The experimental arrangement consisted of simulated, buried cables in two troughs, one filled with sand and the other left under open air conditions. Using repetitive pulses of known amplitude and frequency, measurements at the receiving antenna were used to compare the loss path between the different media (air and soil). Moreover the minimum detectable amplitude of the injected pulse in the soil filed trough was recorded using pulses of varying amplitude but fixed frequency. The experimental arrangement is described in detail in the next section.

\section{EXPERIMENTAL SETUP}

\section{A. Surface Trough Arrangement}

A full sized model of a cable trough has been already constructed just outside the Tony Davies High Voltage Laboratory, in order to serve the purpose of simulating underground power cables. Two identical sections in terms of materials used and dimensions were constructed, with the difference being that one was filled with sand (sand-filled) and the other was left open to air (air-filled). A cross sectional view of the arrangement, together with the dimensions, is given in Fig. 1. The length of each section is around $4 \mathrm{~m}$ (x-coordinate) and the width is $0.6 \mathrm{~m}$ (y-coordinate in a right-handed coordinate system). The depth of both troughs is $0.6 \mathrm{~m}$ (z-coordinate) and the simulated cables were buried at around $0.21 \mathrm{~m}$. The cable was modelled as an aluminium tube (layer 3 in Fig. 1) around which where a heater tape (layer 2 in Fig. 1) was wound. The heater tape and the aluminium tube were wrapped in a foil which was surrounded by a plastic sleeve (layer 1 in Fig. 1), that acts as a moisture barrier and mechanical 


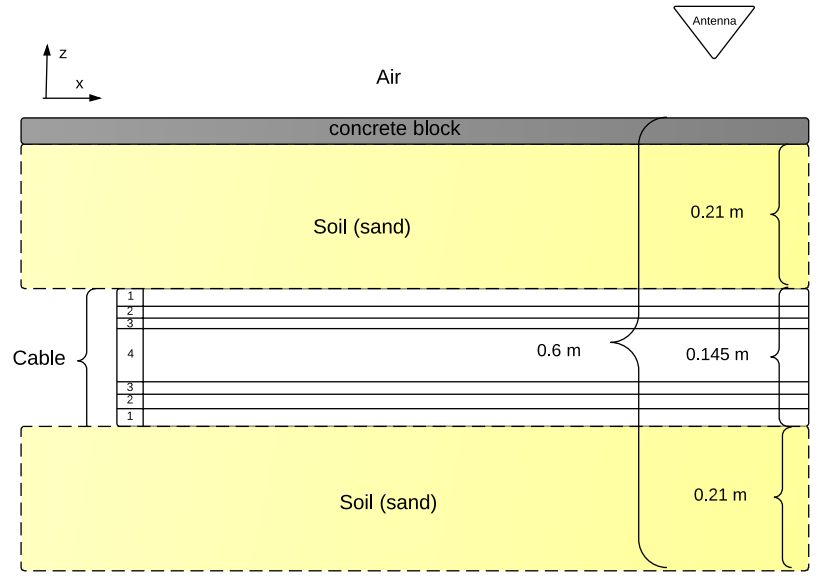

Fig. 1. Cross sectional view of one of the cables sliced through the middle. The layers numbered 1,2, 3 and 4 represent the plastic sleeve, heater tape, aluminium tube and air respectively. The figure is not to scale.

protection for the cable. Each cable trough simulates a three phase circuit in flat arrangement, as shown in Fig. 2. Repetitive signals of varying amplitude and frequency (square waveform) were applied to the heater tape and picked up by two simple antennae, that are described in the next section.

\section{B. Equipment and Antennae Used}

In order to generate repetitive pulses a signal generator was employed (Agilent 81134A) capable of generating square and data burst waveforms up to $3.35 \mathrm{GHz}$. The pulse generator was connected to the heater tape of the cables on either the air or the soil-filled trough. For consistency in all the experiments the pulse was always injected into the middle cable in the trefoil arrangement. To detect the injected pulses two custom-made antennae were connected to a Spectrum Analyser (Rohde \& Schwarz FS315S) able to analyse signals from $9 \mathrm{kHz}$ up to 3.5 $\mathrm{GHz}$. The two antennae used were simple-to-construct designs not optimised to the specifications of the problem but just to

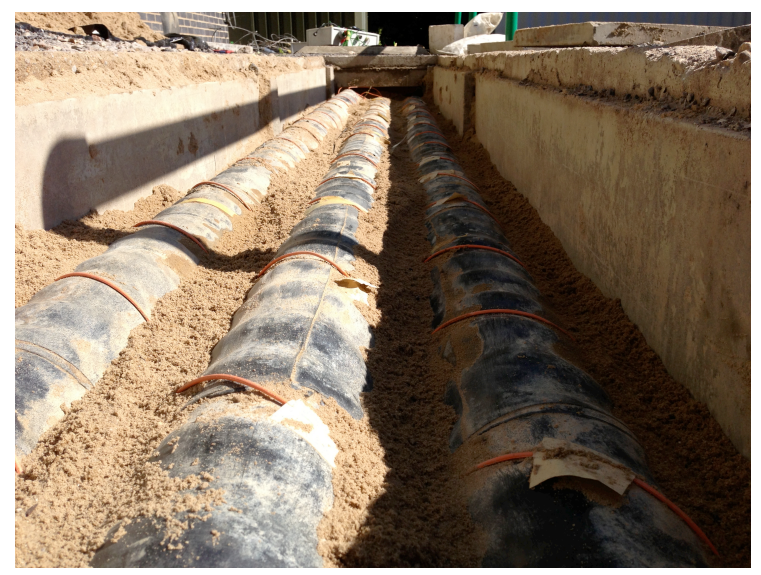

Fig. 2. Actual photograph of the cable trough (the soiled filled one) during the construction phase where the flat arrangement of the cables is shown. prove the concept; a monopole with a height of $1.33 \mathrm{~m}$ and a small loop antenna wound in a circle with a diameter of around $0.3 \mathrm{~m}$.

The monopole antenna was constructed from copper tube of $1.33 \mathrm{~m}$ in height, an external diameter of $1.5 \mathrm{~cm}$ and thickness of the tube was $1 \mathrm{~mm}$. Its predicted resonant point is $\lambda / 4$ where $\lambda$ is the electrical wavelength which can be related to the frequency by

$$
f_{M H z}=\frac{246}{\lambda}
$$

which takes into account the edge effects and the slightly different propagation speed of the electromagnetic wave in the antenna [1]. The theoretical resonant frequency of the monopole was calculated to be around $46 \mathrm{MHz}$, with a purely resistive impedance of around $37 \Omega$. The monopole can also have an anti-resonance, second order resonance and second order anti-resonance at approximately $0.5,0.75$ and 1 wavelengths, respectively. However the actual resonance can vary by up to $30 \%$ depending on the ground plane and which can also have an effect whether the anti resonances and second order effects will be there [2]. The use of a tube instead of a single wire increases the bandwidth, which is related to the ratio of the height to the diameter. The anti-resonance for this antenna occurs at around $92 \mathrm{MHz}$. Simulations for the antenna using MINNEC Pro 1.0.1 showed resonances at around 53.1 MHz (real part of the impedance around $36 \Omega$ ) and anti-resonance around $89.6 \mathrm{MHz}$ (real part of the impedance around $450 \Omega$ ).

The loop antenna belongs to the small loop type of antenna where its diameter $(30 \mathrm{~cm})$ is less than $0.2 \lambda$, which translates to an upper frequency of $164 \mathrm{MHz}$. A small loop antenna relies on magnetic coupling with the source and its difference with a large loop antenna is that, the current is the same throughout the entire loop [1].

\section{THEORY OF PROPAGATION LOSSES}

In this section the theoretical background of the injected path losses from the source to the detection point is presented, identifying the three different regions of operation depending on the distance of the source to the antenna in relation to the wavelength.

\section{A. Far-Field Region}

The propagation path model presented in this section is valid under the assumption that the receiving antenna is in the so called far-field region, where the $\mathrm{E}$ and $\mathrm{H}$ components of the EM wave are in phase but orthogonal to each other and are related by a constant, the intrinsic impedance. If the distance of an electrically small receiving antenna from the source, $r$, is greater than or equal to $3 \lambda$, then it operates in the far-field (or Fraunhofer) region [3]. The propagation of electromagnetic (EM) waves (with the receiving antenna in the far-field) in the soil (and in the subsoil region, $30 \mathrm{~cm}$ to 1 $\mathrm{m}$ below the surface) has already been studied in the context of underground and aboveground communications [4]-[6] for potential applications in agriculture, and underground mines 
[7]. A basic model for underground propagation has been developed in [8] and is based on the Friis equation in free space (ignoring polarization and antenna mismatch losses), given by

$$
P_{r}=P_{t} G_{r} G_{t} \frac{\lambda^{2}}{4 \pi r^{2}}
$$

where $P_{t}$ and $P_{r}$ are the transmitted and received powers, $G_{t}$ and $G_{r}$ the transmit and receive antenna gains. The above equation can be written in logarithmic form as

$$
P_{r}=P_{t}+G_{r}+G_{t}-L_{0}-L_{s}
$$

where $L_{0}$ is the free path loss and $L_{s}$ an additional term to represent the soil losses. The latter can be further analysed by

$$
L_{s}=L_{\beta}+L_{\alpha}
$$

where $L_{\beta}$ is a loss related to the different wavelength $(\lambda)$ of the wave in soil compared to free space $\left(\lambda_{0}\right)$ and $L_{\alpha}$ is due to the soil attenuation. The total loss function $L_{p}\left(L_{0}+L_{s}\right)$ is given by

$$
L_{p}=6.4+20 \log (d)+20 \log (\beta)+8.69 \alpha d
$$

where constant values have been plugged in and $d$ is the distance from the source in meters, $\beta$ is the phase constant given by

$$
\beta=\omega \sqrt{\frac{\mu \epsilon^{\prime}}{2}\left[\sqrt{1+\left[\frac{\epsilon^{\prime \prime}}{\epsilon^{\prime}}\right]^{2}}+1\right]}
$$

in radians $/ \mathrm{m}$ and $\alpha$ is the attenuation loss in meters ${ }^{-1}$ given by

$$
\alpha=\omega \sqrt{\frac{\mu \epsilon^{\prime}}{2}\left[\sqrt{1+\left[\frac{\epsilon^{\prime \prime}}{\epsilon^{\prime}}\right]^{2}}-1\right]}
$$

The parameters $\epsilon^{\prime}$ and $\epsilon^{\prime \prime}$ as well as $\sigma$ can be calculated, for particular frequency ranges, from empirical formulas that involve soil properties [9], [10]. In general the attenuation increases with frequency, volumetric water content [11] and soil composition.

\section{B. Near-Field and Reactive Near-Field Regions}

The region where the receiving antenna operates is related to its distance from the source and can be generally divided into three distinct regions. Depending on whether the antenna is electrically large (its maximum dimension $D$ is much greater than $\lambda$ ) or small ( $D$ much smaller than $\lambda$ ) then different formulas can be employed. The formulas for electrically large antenna can be found in [12]. Given the frequency ranges that are examined here and the antennae designed, they were both classified as electrically small. In this case if the distance falls in the region defined by

$$
3 \lambda>r \geq \frac{\lambda}{2 \pi}
$$

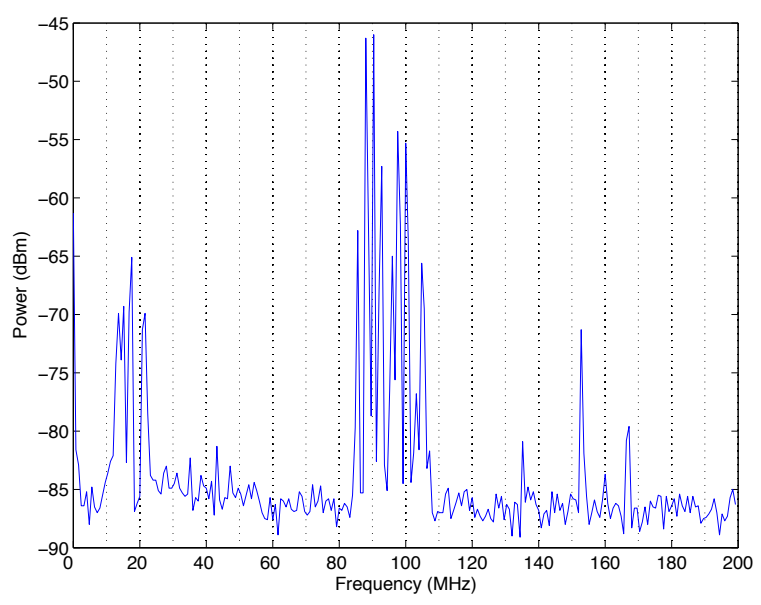

Fig. 3. Spectrum of background noise, from $9 \mathrm{kHz}$ to $200 \mathrm{MHz}$, as picked up by the monopole antenna where areas that should be avoided are clearly seen. The resolution of the spectrum analyser was set to $300 \mathrm{kHZ}$ and the sweep time to $30 \mathrm{~ms}$.

then it operates in the radiating near-field (or Fresnel) region [3]. When the distance criterion

$$
\frac{\lambda}{2 \pi}>r>0
$$

is met the antenna operates in the reactive near-field. For antennae operating in the reactive and Frensel region the free path attenuation is not proportional to $1 / r^{2}$ as given by (2) but also has components of the order of $\left(1 / r^{4}\right.$ and $\left.1 / r^{6}\right)$ as well. The electric and magnetic fields are $90^{\circ}$ out of phase. The analysis becomes a more complicated when in these regions, especially in the reactive zone. For the Frensel region an approximation is to modify the far field loss analysis (equation (5)) by adding another term. This additional term corrects the fact that equation (2) uses the far-field antenna gains $\left(G_{t}\right.$ and $G_{r}$ ), which may vary considerably with distance in the Fresnel region. Therefore the replacement with a distance varying gain $(\mathrm{GFt}(\mathrm{r})$ and $\mathrm{GFr}(\mathrm{r}))$ in (2) can improve the approximations in the near-field region [13].

The frequencies used here were $5 \mathrm{MHz}$ up to $120 \mathrm{MHz}$ so for the two extreme cases the boundaries between the different regions can be calculated using (1). The wavelengths in free space were found to be $49.2 \mathrm{~m}$ and $2.05 \mathrm{~m}$, which indicates an operation between the reactive and near-field region depending on the frequency involved, for an antenna $1 \mathrm{~m}$ from the source. For the soil propagation the wavelength becomes shorter by as much as 4.25 that indicate again an operation between the reactive and near-field region as well. Working in the reactive and near-field needs more careful interpretation of the results as the classical antenna radiation patterns do not hold and the received power can vary significantly with distance.

\section{RESULTS}

Before taking any measurements a recording of the background noise in the vicinity of the cable trough was taken, 
in order to avoid those bands of the frequency spectrum that were already being used. Measurements with both antennae

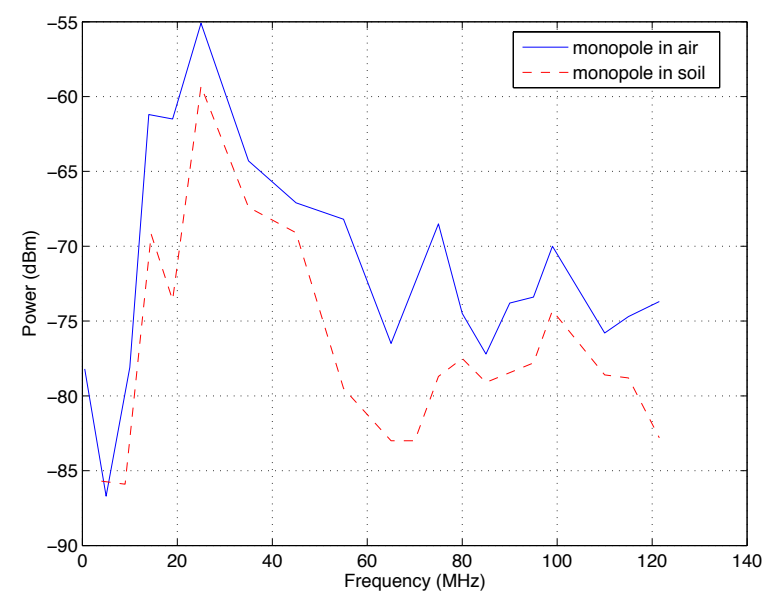

Fig. 4. Plot of the propagation path losses using the monopole antenna for different frequencies. The injected pulse was a square wave of $2 \mathrm{~V}$ peak to peak and $50 \%$ duty cycle.

were performed and the spectrum up to $3.5 \mathrm{GHz}$ was recorded. Taking into account the frequency responses of the antennae used as explained in sectionII-B the frequency range examined was from around $5 \mathrm{MHz}$ to around $120 \mathrm{MHz}$. In this range the first resonance and anti-resonance of the monopole antenna occur, as well as the small loop approximation for the loop antenna is valid. In Fig. 3 the background noise can be seen where the FM radio can be clearly identified in the range from 87.5 to $108 \mathrm{MHz}$. Also other interference regions exist, at around 13.6, 15.8 and 17.6 $\mathrm{MHz}$ as well as some interference around $135.5,155$ and $167 \mathrm{MHz}$.

In order to evaluate the propagation loss path from the signal generator to the measurement antenna pulses were injected at first into the middle cable of the air filled trough. Where possible, every effort was taken to minimise the impedance mismatch between the different components. The injected pulse was a square wave of $50 \%$ duty cycle initially set to a peak to peak value of 2 Volts (measured to be $11.9 \mathrm{dBm}$ on the SA). The frequency was changed to different values covering a range of frequencies from 5 to $120 \mathrm{MHz}$. Special care was taken not to interfere with the identified background noise intervals. The measured responses using both antennae were recorded using a spectrum analyser and the peak value at the frequency of interest was recorded. The monopole antenna showed similar sensitivity to the small loop antenna at most frequencies when measured in air, which can be seen in Fig. 5. The same plot is shown in Fig. 6 for the soil where it can be seen that in general the sensitivity of the monopole is higher but the loop has a flatter bandwidth (especially above $40 \mathrm{MHz}$ ).

Subsequently the pulse was injected into the middle cable of the sand-filled trough and the same procedure was followed where the propagation path loss from the signal generator to

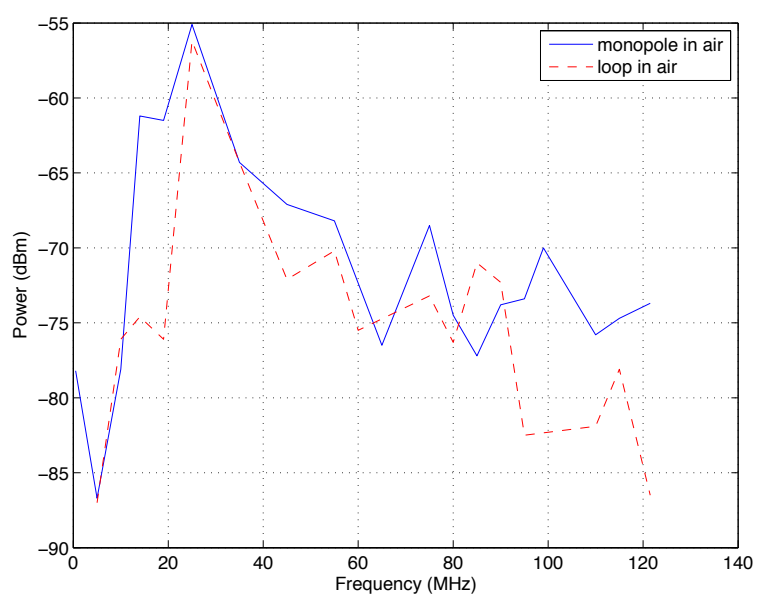

Fig. 5. Plot of the propagation path losses of the monopole antenna against the loop antenna for air propagation at different frequencies. The injected pulse was a square wave of $2 \mathrm{~V}$ peak to peak and $50 \%$ duty cycle.

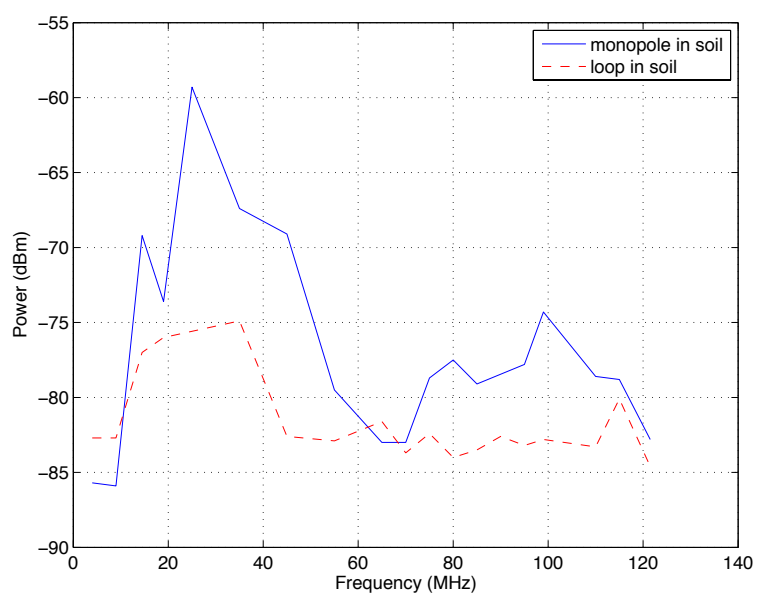

Fig. 6. Plot of the propagation path losses of the monopole antenna against the loop antenna for soil propagation at different frequencies. The injected pulse was a square wave of $2 \mathrm{~V}$ peak to peak and $50 \%$ duty cycle.

the receiving antenna was recorded. The comparison between the propagation path loss between the air and soil filled trough is shown in Fig. 4 for the monopole antenna. The frequencies at which measurements were taken together with the difference (in $\mathrm{dBm}$ ) between the air and soil propagated pulses is shown in Table I.

Next the effect of amplitude change of the source pulse was investigated. The frequency of the pulse was set to $25 \mathrm{MHz}$, which represents the frequency with the lowest path loss based on the analysis in Fig. 4. This provided a best case scenario and resulted in the calculation of the minimum detectable pulse in this specific experimental set-up. The results of this experiment are shown in Fig. 7, from where it can be inferred that the maximum detectable pulse (limit set to $-80 \mathrm{dBm}$ ) was around $200 \mathrm{mV}$ peak to peak. The same procedure was followed for 
TABLE I

DIFFERENCE OF THE AIR VS SOIL PATH LOSS AT THE MEASURED FREQUENCIES

\begin{tabular}{|c|c||c|c||c|c|}
\hline $\begin{array}{c}\text { Frequency } \\
\text { (MHz) }\end{array}$ & $\begin{array}{c}\text { Loss } \\
\text { (dB) }\end{array}$ & $\begin{array}{c}\text { Fre- } \\
\text { quency } \\
\text { (MHz) }\end{array}$ & $\begin{array}{c}\text { Loss } \\
\text { (dB) }\end{array}$ & $\begin{array}{c}\text { Fre- } \\
\text { quency } \\
\text { (MHz) }\end{array}$ & $\begin{array}{c}\text { Loss } \\
\text { (dB) }\end{array}$ \\
\hline 5 & -1 & 45 & 2 & 90 & 5.3 \\
10 & 7.8 & 55 & 11.3 & 95 & 4.4 \\
14 & 8 & 65 & 6.5 & 99 & 4.3 \\
19 & 12.1 & 75 & 14.5 & 110 & 2.8 \\
25 & 4.2 & 80 & 4.2 & 115 & 4.1 \\
\hline
\end{tabular}

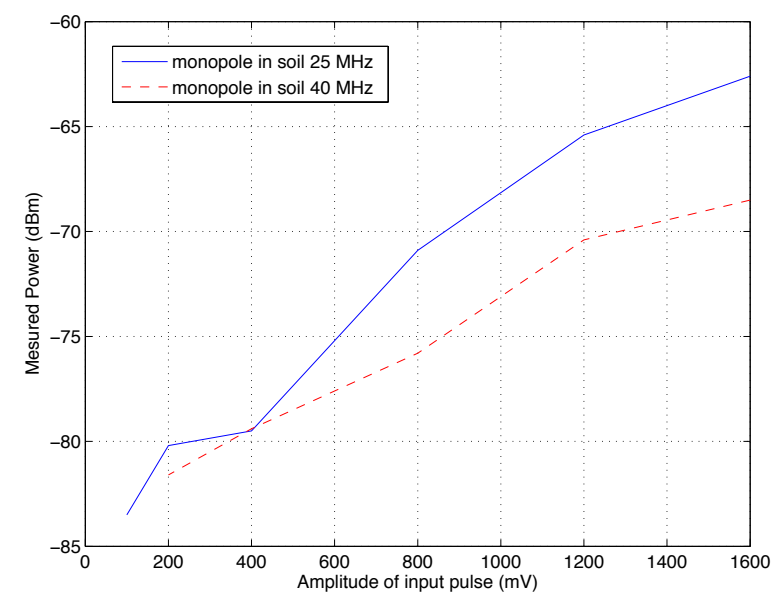

Fig. 7. Detected power at the monopole against the amplitude of the injected signal in the soil filled trough for two different frequencies, $25 \mathrm{MHz}$ and 40 $\mathrm{MHz}$.

$40 \mathrm{MHz}$, which has lower sensitivity (Fig. 4) which provided a maximum detection of around $400 \mathrm{mV}$ peak to peak. Although this can be a realistic amplitude of quite a large PD pulse, the design of the antennae has to be optimised in order to reduce even further the minimum detectable pulse amplitude.

\section{Discussion}

The results presented here gave a preliminary evaluation about the possibility of PD detection in underground cables using aboveground antennae. While the concept was successfully proven using a square wave the main issues that have to be tackled are two. Firstly an antenna with a high bandwidth must be designed which will cover the frequency spectrum from around $10 \mathrm{MHz}$ to around $500 \mathrm{MHz}$. The challenges in the design are the size of the antenna, which has to be a practical one, due to the lower frequency requirement (the wavelength in free space at $10 \mathrm{MHz}$ is 30 metres). The possibility of a combination of antennae or methods of downscaling already employed, such as the inclusion of a dielectric substrate in planar antennae will be explored. The final solution will consist of an array of three antennae, in order to be able to locate the depth as well as the position of the PD. The second issue is the generation actual PDs buried underground in different environmental conditions and soil content and whether they can be detected using the designed antenna. Then using the flat bandwidth antenna improved measurements regarding the attenuation of the propagation through soil can be obtained, as here the fluctuation of the antennae bandwidths masked the accuracy of the results.

\section{CONCLUSION}

In this work a simple experiment was designed based on simulated cables buried under two different trough conditions; one filled with sand while the other left exposed to air. Two simple antennae were designed to pick up signals injected at each cable trough and the path loss characteristics of the air compared to the sand filled one were examined. Moreover as the injected pulses were used to prove the concept that a signal injected underground (as in the case of PD in buried cables and joints) can be picked up by an antenna the amplitude of the maximum detectable pulse was investigated and found to be around $150 \mathrm{mV}$ peak to peak. The prove of the concept that an underground signal generated in buried cables can be detected aboveground using an antenna opens up new research issues which require more experiments and improvement in the design of the antennae in terms of bandwidth. Future work will tackle the issues of an improved antenna design and whether an actual PD source in the ground can be detectable from aboveground.

\section{ACKNOWLEDGMENT}

The authors gratefully acknowledge the financial support by the UK Engineering and Physical Sciences Research Council (EPSRC) under Grant EP/K021699/1.

\section{REFERENCES}

[1] J. Carr, Ed., Practical Antenna Handbook, 4th ed. McGraw-Hil, 2001

[2] M. Weiner, Ed., Monopole Antennas. Marcel Dekke, 2003.

[3] Y. Huang and K. Boyle, Eds., Antennas: From Theory to Practice, 1st ed. New York: John Wiley \& Sons, 2008.

[4] I. F. Akyildiz, Z. Sun, and M. C. Vuran, "Signal propagation techniques for wireless underground communication network," Phys. Commun. (Elsevier), vol. 2, pp. 167-183, September 2009.

[5] A. Silva and M. Vuran, "Communication with aboveground devices in wireless underground sensor networks: An empirical study," in IEEE Int. Conf. on Commun., 2010.

[6] N. Chaamwe, W. Liu, and H. Jiang, "Wave propagation communication models for wireless underground sensor network," in IEEE Int. Conf. on Commun. Technol. (ICCT), 2010, pp. 9-12.

[7] A. Forooshani, S. Bashir, D. Michelson, and S. Noghania, "A survey of wireless communications and propagation modelling in underground mines," IEEE Commun. Surveys Tuts., vol. 15, no. 4, September 2013.

[8] G. Ferrari, Ed., Sensor Networks: Where Theory Meets Practice. Elservier, 2011.

[9] N. A. Peplinski, F. T. Ulaby, and M. C. Dobson, "Dielectric properties of soils in the 0.3-1.3 ghz range," IEEE Trans. Geosci. Remote Sens., vol. 33, no. 3, pp. 803-807, 1995 .

[10] W. Wensink, "Dielectric properties of wet soils in the frequency range 1-3000 MHz," Geophys. Prospect., vol. 41, pp. 671-696, January 1993.

[11] J. O. Curtis, "Moisture effects on the dielectric properties of soils," IEEE Trans. Geosci. Remote Sens., vol. 39, no. 1, pp. 125-128, 2001.

[12] C. Balanis, Ed., Practical Antenna Handbook, 3rd ed. New York: John Wiley \& Sons, 2005.

[13] I. Kim, "Design of a class of antennas utilizing mems, ebg and septum polarizers including near-field coupling analysis," Ph.D. dissertation, UCLA Electrical Engineering, 2012. 